\title{
Screening for broad mite susceptibility in Rhododendron simsii hybrids
}

\author{
Gil Luypaert ${ }^{1, \star}$, Johan Van Huylenbroeck', Jan De Riek' \& Patrick De Clercq² \\ 1 Institute for Agricultural and Fisheries Research (ILVO), Plant Sciences Unit, Applied Genetics and Breeding, Caritasstraat 21, 9090 Melle, \\ Belgium \\ 2 Ghent University, Laboratory of Agrozoology, Department of Crop Protection, Coupure Links 653, 9000 Ghent, Belgium \\ * Corresponding author: gil.luypaert@ilvo.vlaanderen.be
}

Received 12 August 2014, accepted 29 October 2014

\begin{abstract}
Broad mite Polyphagotarsonemus latus, is a key pest of pot azalea (Rhododendron simsii Planch hybrids). No information on variation in susceptibility or potential tolerance within these plants is available to date. We evaluated a selection of 32 Rhododendron cultivars, mainly R. simsii hybrids, for susceptibility to broad mite. The plants were artificially infested in a greenhouse by surrounding each azalea with four broad mite-infested English ivy plants (Hedera helix). Broad mite infestation was evaluated by counting the number of broad mites per shoot tip and assigning a damage rate. Results indicated a comparable infestation rate expressed as the number of mites on all cultivars at the initial stage of the experiments. Correlations between the average damage rate and the number of broad mites per shoot tip on all cultivars at different time intervals were significantly positive, although low, in each experiment, with $R^{2}$-values of 0.14 and 0.61 . At the end of the experiments significant differences in susceptibility between the evaluated cultivars were observed. The cultivars, 'Emil De Coninck' and 'Mont Blanc' were rated as the most susceptible, whereas 'Mistral' and its bud sport 'Elien' were tolerant towards the broad mite, because damage rates were low and very few broad mites were found.
\end{abstract}

Key words: Azalea, Polyphagotarsonemus latus, genetic variation, tolerance

\section{Introduction}

The broad mite, Polyphagotarsonemus latus (Acari: Tarsonemidae), is a cosmopolitan pest affecting numerous plants. It causes severe damage to many economically important agricultural crops and ornamental plants worldwide (Jeppson et al. 1975, Gerson 1992, Zhang 2003). The broad mite is usually found on the lower surfaces of young apical leaves and in flowers, where it deposits its eggs (Gerson 1992). Although broad mites feed mainly on the epidermis of the plant parts, structural aberrations occur throughout the tissue (Grinberg et al. 2005). The pest feeds on the youngest growth tips, causing malformation of the flower buds and the terminal leaves. The latter become severely stunted and hardened, curl down at the edges, and show brownish or reddish lower surfaces (Łabanowski \& Soika 2006). Ewing
(1939) was the first to report the occurrence of broad mites on Ericaceae in North America. According to Navajas et al. (2010), broad mites were first recorded in Italy in 1961. Since then, the mite has been found in other European countries including Belgium, where it was reported for the first time on R. simsii in the mid-1980 s (Heungens 1986). During recent years, a significant increase in the pest status of broad mites in pot azalea has been observed, mainly due to restrictions on the use of broad spectrum pesticides, including the complete prohibition of the use of endosulfan (an active component in many plant protection products) from July 2007 onwards (EU directive 2005/864/EC). Integrated pest management (IPM) and its EU-wide implementation (EU directive 2009/128/EC) forces growers to develop sustainable strategies for the control of this pest (Gobin et al. 2011, Gobin et al. 2013). In an IPM based strategy, preventative cultural practices are essential during the growing process. One of these measures could be developing and growing tolerant $R$. simsii cultivars.

Tolerant genotypes to broad mite have been successfully selected in various crops. Most studies related to screening for susceptibility or to factors leading to tolerance against broad mite have been performed on Capsicum spp. Matos et al. (2009) found that trichomes act as a limiting trait for the development of broad mites, especially when the trichomes occur in higher densities and are well-distributed on the leaf surface. According to Thungrabeab \& Boonlertnirun (2002), trichome density and leaf thickness tend to be predominant factors related to resistance (few mites and low damage rates) to broad mite in Capsicum annuum (line P 3066-20-20 and line P 3067-17-2) and C. frutescens (line CHMC 1118). For both host species, Echer et al. (2002) concluded that symptom assessment was a more suitable method for determining resistance of genotypes than counting the number of adult mites present on the plant. This resulted from an indirect relation between the number of broad mites and the intensity of the damage symptoms observed on certain genotypes (Echer et al. 2002). Furthermore, according to Basset (1981), the manifestation of symptoms can quickly evolve in certain host plants, where a small number of mites would be enough to cause economic damage. Susceptible as well as resistant accessions were identified in screenings of $C$. annuum cultivars using a natural infestation of broad mites (Ahmed et al. 2001, Desai et al. 2006, Desai et al. 2007, Kulkarni et al. 2011). The correlation of biochemical constituents in 15 C. annuum cultivars with mite incidence was assessed by 
Ahmed et al. (2001). Based on a study on the relationship of broad mites to host phenology and injury levels in $C$. annuum, de Coss-Romero \& Peña (1998) concluded that damage caused by broad mites appears to depend on the stage of development of $C$. annuum. The plant is most vulnerable to infestation during the vegetative growth phase (Rodríguez et al. 2011). Vichitbandha \& Chandrapatya (2011) also studied the effect of broad mites on damage and yield in C. annuum 'Tavi 64' and showed significant yield reduction after a plant damage level of more than 50\% curled shoots. Reports on screening for resistance against broad mite in other crops are also available, e.g. potato species Solanum polyadenium, S. tarijense and S. berthaultii (Gibson \& Valencia 1978), eggplant (Solanum melongena) (Lianyou et al. 1999), watermelon (Cirtullus lanatus var. lanatus) (Kousik et al. 2007) and jute (Corchorus spp.) (Jana et al. 2007). To our knowledge no previous studies have assessed genetic variance in susceptibility to broad mite in ornamental plants.

The main objective of the present study was to evaluate the genetic variation of a selection of $R$. simsii hybrids in terms of susceptibility to broad mites. The use of cultivars tolerant to pests is sustainable and fits well into an overall IPM strategy for broad mite control. Furthermore, information presented in this article will be useful for breeders for the development of new tolerant cultivars.

\section{Materials and methods}

\section{Plant material}

A total of 32 Rhododendron cultivars were collected. Thirty belong to the subgenus Tsutsusi, section Tsutsusi; two accessions ( $R$. burmanicum (Yellow) and 'Fragrantissimum') belong to the subgenus Rhododendron, section Rhododendron, subsection Maddenia (Table 1). Cuttings originated from a core collection of Rhododendron spp. kept at ILVO, Melle, Belgium. Plants were grown from rooted cuttings and pruned once. This material was used in experiment 1 or experiment 2 , six or eight weeks after regrowth. For experiment 2, cultivars 'Bergmann Feu', 'Doctor Bergmann Albus' and 'Madame Charles Gabert' were grafted onto rootstock of R. scabrum hybrid 'Concinna'. In experiment 3 , plants used were cut three times before being used in the experiment. Before the experiments plants were grown in a standard greenhouse under natural light conditions with a minimum temperature of $5^{\circ} \mathrm{C}$ and ventilation temperature set at $20^{\circ} \mathrm{C}$.

\section{Infestation technique}

A stock culture of broad mites was maintained on Hedera helix, initiated from naturally infested $H$. helix 'Montgomory' and 'Chester' plants. For this purpose the plants were grown in a greenhouse with night/day temperature set at $17 / 20^{\circ} \mathrm{C}$. The photoperiod was 16:8 h (L:D). When light intensity was below $150 \mathrm{~W} \mathrm{~m}^{-2}$, additional assimilation light $\left(50 \mu \mathrm{mol} \mathrm{m}^{-2}\right.$ $\mathrm{s}^{-1}$ ) was provided using Gavita GAN 600 AL fixtures (GAVITA Nederland B.V., the Netherlands) with 600W 230V lamps.
In each experiment, the Rhododendron cultivars were artificially infested by surrounding each individual plant with four $H$. helix plants containing a stock culture of broad mites. All $H$. helix plants were selected after visual inspection of mite damage. Five $H$. helix samples were examined to confirm pest presence, using an observation microscope (Leica MZ6, Germany). Greenhouse conditions were set at $21^{\circ} \mathrm{C}$ as a minimum temperature and ventilation was activated at $26^{\circ} \mathrm{C}$ during all experiments, and the photoperiod was 16:8 $\mathrm{h}$ (L:D). When light intensity was below $100 \mathrm{~W} \mathrm{~m}^{-2}$, additional assimilation light $\left(100 \mu \mathrm{mol} \mathrm{m} \mathrm{m}^{-2} \mathrm{~s}^{-1}\right)$ was provided by a conventional fixture, HS2000 (Hortilux Schréder B.V., the Netherlands) using Master GreenPower 600W 400V lamps (Philips, the Netherlands). Above $200 \mathrm{~W} \mathrm{~m}^{-2}$, shades were used to reduce the light intensity. Light intensity was measured with LP02 pyranometer (Hukseflux Thermal Sensors B.V., the Netherlands) connected to a logging system.

\section{Scoring of cultivars and counting of broad mites}

Broad mite effect on Rhododendron cultivars was rated according to the symptoms. A scoring system of five classes was used, where 0 = no injury observed; 1 = very low injury, very slight browning of the youngest leaves of one or more shoot tips, less than $25 \%$ of the shoot tips showing symptoms; 2 = slight injury, all shoot tips show browning and curling of youngest leaves; symptoms are visible on $26-50 \%$ of the plant; 3 = moderate injury, some shoot tips bronzed and deformed, mature leaves start curling, between 51 and $75 \%$ of the plant is injured; and $4=$ severe injury, all shoot tips bronzed, hard and necrotic and mature leaves severely distorted, more than $75 \%$ of the plant shows damage symptoms.

Quantification of broad mites was assayed on the apical shoot tips (cut off between the third and fourth free-standing leaf as counted, starting from the apex). Samples were collected in a plastic vial with screw cap $(60 \mathrm{ml}$, diameter $33 \mathrm{~mm}$ ) (Gosselin, France) filled with $20 \mathrm{ml}$ ethanol 70\%. The vial was shaken and its contents poured over a nylon sieve (mesh size $1.5 \mathrm{~mm}$ ), on a reusable filter holder with receiver (Nalge Nunc International, NY, USA), equipped with a cellulose mixed ester type Machery-Nagel Porafil ${ }^{\circledR}$ CM filter (pore size $0.45 \mu \mathrm{m}$, diameter $47 \mathrm{~mm}$, black and with white grid). Using a vacuum pump, the membrane filter was drained and then transferred into a $60 \mathrm{~mm}$ diameter petri dish (Greiner Bio One BVBA/SPRL, Belgium) for counting under an observation microscope. In experiments 1 and 2 , all life stages were counted as one, to facilitate the counting process. During experiment 3, eggs, larvae, nymphs, males and females were recorded separately in order to evaluate the population dynamics of the mite.

\section{Experiment 1}

Thirty cultivars (01-14-1 and NA40246 KURIO were excluded) were screened in four replicated plots from November 2012 till January 2013. Each of the four plots contained one entry 
Table 1: Selection of Rhododendron cultivars used in screening experiments for broad mite susceptibility. For each of the selected cultivars the species name and the parentage are given. This table also indicates in which of the experiments each cultivar was included.

\begin{tabular}{|c|c|c|c|c|c|}
\hline Cultivar & Species & Parentage ${ }^{a}$ & Exp. 1 & Exp. 2 & Exp. 3 \\
\hline 01-14-1 & R. simsii hybrid & - & & $x$ & \\
\hline $95-20-5$ & R. simsii hybrid & - & $x$ & & \\
\hline Aiko Pink & R. simsii hybrid & - & $x$ & $x$ & $x$ \\
\hline Antartica & R. simsii hybrid & $\begin{array}{l}\text { Reported to be derived from eriocarpum crossed } \\
\text { with various simsii cultivars }\end{array}$ & $x$ & $x$ & \\
\hline Bergmann Feu & R. simsii hybrid & Sport from ‘Docteur Bergmann’ & $x$ & $x$ & \\
\hline Christine Matton & R. simsii hybrid & Selection from simsii & $x$ & $x$ & \\
\hline Docteur Bergmann Albus & R. simsii hybrid & Sport of the 'Docteur Bergmann' series & $x$ & $x$ & \\
\hline Elien & R. simsii hybrid & Sport from 'Mistral’^ & $x$ & $x$ & $x$ \\
\hline Emil De Coninck & R. simsii hybrid & Sport from 'Jean Haerens' & $x$ & $x$ & \\
\hline Kassandra & R. simsii hybrid & - & $x$ & $x$ & \\
\hline Lara & R. simsii hybrid & ‘Memoria Sander’ (s) X ‘Heiwa-no-hikari’ & $x$ & $x$ & \\
\hline Madame Charles Gabert & R. simsii hybrid & Sport from ‘Docteur Bergmann Saumoneus' & $x$ & $x$ & \\
\hline Mevrouw Gerard Kint & R. simsii hybrid & Sport from ‘Glaser Nummer 10’* & $x$ & $x$ & $x$ \\
\hline Mevrouw Marc Van Eetvelde & R. simsii hybrid & 'Pink Dream’ X ‘Heiwa-no-hikari’* & $x$ & $x$ & \\
\hline Michelle Marie & R. simsii hybrid & ‘Rosalie’ X un-named seedling & $x$ & $x$ & $x$ \\
\hline Mistral & R. simsii hybrid & ‘Osaka’ X’Heiwa-no-hikari’ & $x$ & $x$ & \\
\hline Mont Blanc & R. simsii hybrid & - & $x$ & $x$ & \\
\hline Noralinde & R. simsii hybrid & - & $x$ & $x$ & \\
\hline Nordlicht & R. simsii hybrid & Sport from 'Hellmut Vogel' & $x$ & $x$ & $x$ \\
\hline Otto & R. simsii hybrid & simsii derivative & $x$ & $x$ & \\
\hline Princess Maria Pia & R. simsii hybrid & 'Tempérance' X ‘Ambrosius Superbus' & $x$ & $x$ & \\
\hline Rosa Belton & R. simsii hybrid & Sport from 'James Belton’ & $x$ & & \\
\hline Roxette & R. simsii hybrid & - & $x$ & $x$ & \\
\hline Sachsenstern & R. simsii hybrid & - & $x$ & $x$ & $x$ \\
\hline Tamira & R. simsii hybrid & - & $x$ & $x$ & \\
\hline Thesla & R. simsii hybrid & 'Sankt Valentin' X 'Mevrouw Gerard Kint' & $x$ & $x$ & \\
\hline Yankadi & R. simsii hybrid & - & $x$ & $x$ & \\
\hline Desiree & R. obtusum hybrid & ‘Ambrosiana’ X 'Jubile’ (Japanese azalea)* & $x$ & $x$ & \\
\hline Nazarena & R. obtusum hybrid & ‘Ambrosiana' X 'Jubile’ (Japanese azalea)* & $x$ & $x$ & \\
\hline Fragrantissimum & Rhododendron hybrid & - & $x$ & $x$ & \\
\hline R. burmanicum (Yellow) & R. burmanicum & - & $x$ & $x$ & \\
\hline NA 40246 KURIO & R. tamurae & - & & $x$ & \\
\hline
\end{tabular}

a Data based on Leslie (2004) unless indicated by * data were collected from Heursel (1999).

of a cultivar. Three and seven weeks post infestation, a single plot (three shoot tips per plant) was used for quantitative detection. Visual scoring of each plant was done weekly for seven consecutive weeks.

\section{Experiment 2}

Three replicated plots were used to screen 30 cultivars (95-20-5 and 'Rosa Belton' were excluded). Each plot contained three entries of a single cultivar, resulting in a total of nine replications per cultivar. During the six-week period between March 2013 and April 2013, all plants were scored weekly for damage symptoms. Broad mites were quantified 2, 4 and 6 weeks post infection by harvesting marked shoot tips. These tips were labelled prior to the start of the experiment in order to preclude bias during subsequent sampling. A single entry from each cultivar was sampled within each plot at every time interval.

\section{Experiment 3}

The cultivars 3, 'Aiko Pink', 'Elien', 'Mevrouw Gerard Kint', 'Michelle Marie', 'Nordlicht' and 'Sachsenstern' were screened in six individual plots. Each plot consisted of nine plants and was separated from the others by one meter of free space. Twelve days after the initial infestation was set up, re-infestation was done by placing new infested $H$. helix plants, because the first infestation was ineffective (no visual damage 
symptoms). Therefore plant damage symptoms were scored weekly during an eight-week period between October 2013 and November 2013. Broad mites were quantified every two weeks on each entry. Shoot tips used for quantitative detection were again labeled before broad mite infection began.

\section{Statistical analysis}

Two-way analyses of variance (ANOVA) (IBM SPSS Statistics; version 22) were conducted to evaluate whether the cultivars had a different effect on broad mite counts, when sampled at different time intervals in experiment 1 or 2 . Likewise, damage rates were analyzed using two-way-ANOVA. Pearson correlations were calculated between the average number of broad mites per shoot tip and the disease rate for each cultivar in experiment 1 and 2 . A repeated measures ANOVA was used to evaluate broad mite counts or damage rates at different time intervals for each cultivar in experiment 3. Degrees of freedom were corrected using the Greenhouse-Geisser correction when Mauchly's test of sphericity showed to be violated. In case of a significant effect, means were separated using Bonferroni (damage rate) or LSD correction (broad mite counts). Broad mite counts were log transformed $(\ln (x+1))$ and square root transformations were applied for damage rates before the statistical analyses.

\section{Results}

In experiment 1 , broad mites were retrieved after three weeks on plants of all cultivars, except for 'Elien' (Table 2).

Table 2: Average damage rates and broad mite counts of 30 selected Rhododendron cultivars at seven time intervals post infestation (dpi) in experiment 1 . Average broad mite counts are given in absolute numbers. Data are means $\pm \mathrm{SE}$. $\mathrm{n}=3$ for broad mite counts, and $n=4$ (7, 14 and $21 \mathrm{dpi})$ or $n=3(28,35,42$ and $49 \mathrm{dpi})$ for the damage rates.

\begin{tabular}{|c|c|c|c|c|c|c|c|c|c|}
\hline \multirow{2}{*}{ Cultivar } & \multicolumn{7}{|c|}{ Damage rates } & \multicolumn{2}{|c|}{ Broad mites } \\
\hline & $7 \mathrm{dpi}$ & $14 \mathrm{dpi}$ & $21 \mathrm{dpi}$ & $28 \mathrm{dpi}$ & $35 \mathrm{dpi}$ & $42 \mathrm{dpi}$ & $49 \mathrm{dpi}$ & $21 \mathrm{dpi}$ & $49 \mathrm{dpi}$ \\
\hline $95-20-5$ & 0 & 0 & $0.5 \pm 0.3$ & $0.3 \pm 0.3$ & $1.0 \pm 0.0$ & $1.3 \pm 0.3$ & $1.7 \pm 0.3$ & $7.3 \pm 4.7$ & $71.0 \pm 29.2$ \\
\hline Aiko Pink & 0 & 0 & $1.3 \pm 0.3$ & $2.0 \pm 0.6$ & $2.0 \pm 0.6$ & $2.3 \pm 0.7$ & $2.3 \pm 0.7$ & $11.3 \pm 2.4$ & $11.6 \pm 1.7$ \\
\hline Antartica & 0 & 0 & $0.5 \pm 0.3$ & $1.0 \pm 0.6$ & $1.7 \pm 0.3$ & $2.0 \pm 0.6$ & $2.0 \pm 0.6$ & $19.6 \pm 9.0$ & $13.6 \pm 4.9$ \\
\hline Bergmann Feu & 0 & $0.5 \pm 0.3$ & $1.0 \pm 0.4$ & $1.7 \pm 0.3$ & $2.0 \pm 0.0$ & $2.3 \pm 0.3$ & $2.7 \pm 0.3$ & $0.3 \pm 0.3$ & $14.0 \pm 5.3$ \\
\hline Christine Matton & $0.3 \pm 0.3$ & $0.8 \pm 0.3$ & $2.0 \pm 0.0$ & $2.0 \pm 0.6$ & $2.3 \pm 0.3$ & $2.3 \pm 0.3$ & $2.3 \pm 0.3$ & $6.7 \pm 3.2$ & $21.3 \pm 5.8$ \\
\hline Doctor Bergmann Albus & 0 & 0 & $1.3 \pm 0.3$ & $1.3 \pm 0.3$ & $2.3 \pm 0.3$ & $2.3 \pm 0.3$ & $2.7 \pm 0.3$ & $8.3 \pm 2.9$ & $16.6 \pm 3.5^{\mathrm{a}}$ \\
\hline Elien & 0 & 0 & 0 & 0 & 0 & $0.3 \pm 0.3$ & $0.3 \pm 0.3$ & 0 & $3.3 \pm 2.0$ \\
\hline Emil De Coninck & 0 & 0 & $0.8 \pm 0.5$ & $2.0 \pm 0.6$ & $1.7 \pm 0.3$ & $2.3 \pm 0.3$ & $3.0 \pm 0.6$ & $20.0 \pm 3.6$ & $57.6 \pm 23.5$ \\
\hline Kassandra & 0 & $0.3 \pm 0.3$ & $0.8 \pm 0.5$ & $1.3 \pm 0.3$ & $1.3 \pm 0.3$ & $2.0 \pm 0.6$ & $2.0 \pm 0.6$ & $1.3 \pm 0.9$ & $7.3 \pm 3.0$ \\
\hline Lara & 0 & 0 & $0.3 \pm 0.3$ & 0 & 0 & $0.3 \pm 0.3$ & $0.7 \pm 0.3$ & $0.3 \pm 0.3$ & $8.3 \pm 4.9$ \\
\hline Madame Charles Gabert & 0 & $0.5 \pm 0.3$ & $1.3 \pm 0.5$ & $1.7 \pm 0.3$ & $2.0 \pm 0.0$ & $2.7 \pm 0.3$ & $2.7 \pm 0.3$ & $10.0 \pm 4.0$ & $18.3 \pm 1.5$ \\
\hline Mevrouw Gerard Kint & 0 & 0 & $0.5 \pm 0.3$ & $1.3 \pm 0.3$ & $1.3 \pm 0.3$ & $1.3 \pm 0.3$ & $1.3 \pm 0.3$ & $9.7 \pm 2.0$ & $9.0 \pm 0.6$ \\
\hline Mevrouw Marc Van Eetvelde & 0 & 0 & 0 & 0 & 0 & $0.7 \pm 0.3$ & $0.7 \pm 0.3$ & $2.0 \pm 2.0$ & $7.7 \pm 5.7$ \\
\hline Michelle Marie & 0 & $0.5 \pm 0.3$ & $1.3 \pm 0.3$ & $1.0 \pm 0.0$ & $1.0 \pm 0.0$ & $2.3 \pm 0.7$ & $2.3 \pm 0.7$ & $31.6 \pm 7.5$ & $13.6 \pm 5.7$ \\
\hline Mistral & 0 & 0 & 0 & 0 & 0 & 0 & 0 & $0.3 \pm 0.3$ & $6.3 \pm 3.2$ \\
\hline Mont Blanc & 0 & $0.3 \pm 0.3$ & $1.3 \pm 0.6$ & $2.0 \pm 0.6$ & $2.0 \pm 0.6$ & $3.0 \pm 0.6$ & $3.0 \pm 0.6$ & $8.7 \pm 2.3$ & $10.0 \pm 6.0$ \\
\hline Noralinde & 0 & 0 & $1.0 \pm 0.4$ & $1.3 \pm 0.3$ & $1.7 \pm 0.3$ & $2.0 \pm 0.6$ & $2.3 \pm 0.7$ & $9.0 \pm 3.6$ & $26.6 \pm 3.3$ \\
\hline Nordlicht & 0 & $0.5 \pm 0.3$ & $0.5 \pm 0.3$ & $1.7 \pm 0.7$ & $1.7 \pm 0.7$ & $2.3 \pm 0.7$ & $2.3 \pm 0.7$ & $2.0 \pm 2.0$ & $10.6 \pm 2.2$ \\
\hline Otto & 0 & $0.5 \pm 0.3$ & $1.5 \pm 0.5$ & $2.0 \pm 0.6$ & $2.0 \pm 0.6$ & $2.3 \pm 0.7$ & $2.3 \pm 0.7$ & $13.3 \pm 2.7$ & $10.6 \pm 4.8$ \\
\hline Prinses Maria Pia & 0 & 0 & 0 & $0.3 \pm 0.3$ & $1.0 \pm 0.0$ & $1.0 \pm 0.0$ & $1.3 \pm 0.3$ & $3.3 \pm 1.9$ & $16.0 \pm 12.0^{a}$ \\
\hline Rosa Belton & 0 & 0 & $1.0 \pm 0.0$ & $1.3 \pm 0.3$ & $2.3 \pm 0.3$ & $2.3 \pm 0.3$ & $2.0 \pm 0.6$ & $6.0 \pm 2.3$ & $25.0 \pm 6.0$ \\
\hline Roxette & 0 & $0.3 \pm 0.3$ & $1.0 \pm 0.4$ & $1.0 \pm 0.6$ & $1.7 \pm 0.7$ & $2.0 \pm 0.6$ & $2.3 \pm 0.7$ & $67.5 \pm 31.5^{a}$ & $20.0 \pm 10.0$ \\
\hline Sachsenstern & 0 & 0 & $0.5 \pm 0.3$ & $1.3 \pm 0.3$ & $1.0 \pm 0.0$ & $1.3 \pm 0.3$ & $1.3 \pm 0.3$ & $8.7 \pm 4.3$ & $9.0 \pm 2.0^{\mathrm{a}}$ \\
\hline Tamira & 0 & 0 & $1.0 \pm 0.4$ & $1.3 \pm 0.3$ & $2.0 \pm 0.6$ & $2.0 \pm 0.6$ & $2.0 \pm 0.6$ & $42.5 \pm 6.5^{a}$ & $25.0 \pm 5.1$ \\
\hline Thesla & 0 & 0 & $0.3 \pm 0.3$ & $1.0 \pm 0.0$ & $1.3 \pm 0.3$ & $1.3 \pm 0.3$ & $1.3 \pm 0.3$ & $9.0 \pm 2.1$ & $3.7 \pm 2.2$ \\
\hline Yankadi & 0 & $0.3 \pm 0.3$ & $1.0 \pm 0.4$ & $1.3 \pm 0.3$ & $1.7 \pm 0.3$ & $2.3 \pm 0.7$ & $2.3 \pm 0.7$ & $33.6 \pm 11.1$ & $61.6 \pm 6.7$ \\
\hline Desiree & 0 & 0 & $0.5 \pm 0.3$ & $1.3 \pm 0.3$ & $1.3 \pm 0.3$ & $2.0 \pm 0.6$ & $2.0 \pm 0.6$ & $22.6 \pm 3.18$ & $15.3 \pm 3.2$ \\
\hline Nazarena & 0 & 0 & 0 & $0.7 \pm 0.3$ & $0.7 \pm 0.3$ & $1.0 \pm 0.0$ & $1.3 \pm 0.3$ & $5.3 \pm 0.3$ & $4.7 \pm 0.9$ \\
\hline Fragrantissimum & 0 & 0 & $0.3 \pm 0.3$ & 0 & $0.3 \pm 0.3$ & $0.3 \pm 0.3$ & $0.7 \pm 0.3$ & $3.5 \pm 0.5^{a}$ & $9^{b}$ \\
\hline R. burmanicum (Yellow) & 0 & 0 & 0 & $1.3 \pm 0.3$ & $1.7 \pm 0.3$ & $1.3 \pm 0.3$ & $1.3 \pm 0.3$ & $20.3 \pm 10.4$ & $4.0 \pm 2.1$ \\
\hline
\end{tabular}

a Average number of broad mites per shoot tip based on two samples.

b Number of broad mites per shoot tip based on one sample. 
Broad mites were recorded on all cultivars after two weeks in experiment 2 (Table 3 ). In experiment 1 , the highest numbers of broad mites was found on 'Roxette' at 21 days post infestation (dpi) and on 95-20-5 at 49 dpi (Table 2). At the end of the experiment, the lowest average number of broad mites was counted on 'Elien'. Both 'Mistral' and its bud sport 'Elien', had the lowest damage rates in experiment 1. At $49 \mathrm{dpi}$, the average rates were 0 and 0.3 for 'Mistral' and 'Elien', respectively. The highest damage rates were recorded for 'Emil De Coninck' and 'Mont Blanc' (Table 2). In experiment 2 , the lowest numbers of broad mites were observed on 'Mistral' at $15 \mathrm{dpi}$ and $29 \mathrm{dpi}$ and on 'Elien' at $43 \mathrm{dpi}$. The highest numbers of broad mites were observed on 'Lara' at 15 dpi and 29 dpi and on 'Emil De Coninck' at 43 dpi (Table 3). In experiment 2, 'Elien', 'Fragrantissimum' and 'Mistral' had the lowest average damage rates $(\leq 0.6)$.
The highest damage rates were recorded for 'Emil De Coninck', 'Mont Blanc' and 'Otto', ranging between 3.3 and 3.7 (Table 3). For both experiments a significant interaction was found between time interval and cultivar for the number of broad mites and the given damage rates (Table 4). The correlations $\left(R^{2}\right)$ between the average damage rates and broad mite counts in experiment 1 where 0.21 and 0.36 at 21 and $49 \mathrm{dpi}$, respectively. In experiment $2, R^{2}$-values were $0.25,0.61$ and 0.14 at 15, 29 and 43 dpi, respectively. At every time of recording, these correlations were found to be significant $(p<0.05)$.

Finally, a third experiment was conducted to evaluate broad mite infestation on six selected cultivars when the mite's feeding choices were limited between a single selected cultivar and $H$. helix. Therefore each cultivar was screened in an individual plot (experiment 3). The selection of 'Aiko

Table 3: Average damage rates and broad mite counts of 30 selected Rhododendron cultivars at six time intervals post infestation (dpi) in experiment 2 . Average broad mite counts are given in absolute numbers. Data are means $\pm S E$. $n=3$ for broad mite counts, and $n=9$ for the damage rates.

\begin{tabular}{|c|c|c|c|c|c|c|c|c|c|}
\hline \multirow{2}{*}{ Cultivar } & \multicolumn{6}{|c|}{ Damage rate } & \multicolumn{3}{|c|}{ Broad mites } \\
\hline & $8 \mathrm{dpi}$ & $15 \mathrm{dpi}$ & $22 \mathrm{dpi}$ & $29 \mathrm{dpi}$ & $36 \mathrm{dpi}$ & $43 \mathrm{dpi}$ & $15 \mathrm{dpi}$ & $29 \mathrm{dpi}$ & $43 \mathrm{dpi}$ \\
\hline 01-14-1 & 0 & $0.9 \pm 0.1$ & $1.0 \pm 0.0$ & $1.5 \pm 0.2$ & $2.0 \pm 0.2$ & $2.5 \pm 0.2$ & $17.3 \pm 5.0$ & $13.0 \pm 4.0$ & $16.0 \pm 3.8$ \\
\hline Aiko Pink & 0 & $1.8 \pm 0.2$ & $2.6 \pm 0.2$ & $2.9 \pm 0.1$ & $3.0 \pm 0.0$ & $3.0 \pm 0.0$ & $15.0 \pm 5.7$ & $25.6 \pm 11.6$ & $51.6 \pm 5.4$ \\
\hline Antartica & $0.2 \pm 0.2$ & $1.2 \pm 0.2$ & $2.3 \pm 0.2$ & $2.6 \pm 0.2$ & $2.9 \pm 0.1$ & $3.0 \pm 0.0$ & $37.0 \pm 4.2$ & $29.0 \pm 6.5$ & $56.6 \pm 15.1$ \\
\hline Bergmann Feu & 0 & $1.2 \pm 0.2$ & $2.0 \pm 0.2$ & $2.6 \pm 0.2$ & $2.4 \pm 0.2$ & $2.8 \pm 0.2$ & $37.0 \pm 4.0$ & $32.0 \pm 6.4$ & $35.3 \pm 15.2$ \\
\hline Christine Matton & $1.0 \pm 0.3$ & $1.9 \pm 0.3$ & $2.7 \pm 0.2$ & $2.8 \pm 0.2$ & $2.9 \pm 0.1$ & $3.0 \pm 0.0$ & $17.6 \pm 4.7$ & $27.0 \pm 3.5$ & $19.0 \pm 5.2$ \\
\hline Doctor Bergmann Albus & $0.2 \pm 0.2$ & $1.3 \pm 0.2$ & $2.0 \pm 0.0$ & $2.9 \pm 0.1$ & $2.9 \pm 0.1$ & $3.0 \pm 0.0$ & $21.3 \pm 4.6$ & $9.7 \pm 5.8$ & $13.0 \pm 4.7$ \\
\hline Elien & 0 & 0 & 0 & 0 & $0.1 \pm 0.1$ & $0.2 \pm 0.2$ & $13.0 \pm 12.0$ & $4.0 \pm 4.0$ & $2.0 \pm 0.6$ \\
\hline Emil De Coninck & $0.7 \pm 0.2$ & $1.9 \pm 0.3$ & $2.9 \pm 0.1$ & $3.0 \pm 0.0$ & $3.3 \pm 0.2$ & $3.3 \pm 0.2$ & $64.6 \pm 46.3$ & $77.0 \pm 22.6$ & $131.0 \pm 43.7$ \\
\hline Kassandra & 0 & $1.3 \pm 0.2$ & $2.1 \pm 0.1$ & $2.7 \pm 0.2$ & $3.0 \pm 0.0$ & $3.1 \pm 0.1$ & $22.0 \pm 10.1$ & $13.3 \pm 2.3$ & $42.6 \pm 2.6$ \\
\hline Lara & 0 & $1.3 \pm 0.2$ & $2.0 \pm 0.3$ & $2.6 \pm 0.3$ & $2.7 \pm 0.2$ & $2.7 \pm 0.2$ & $160.0 \pm 30.0$ & $130.0 \pm 66.2$ & $40.0 \pm 6.1$ \\
\hline Madame Charles Gabert & $0.4 \pm 0.2$ & $1.7 \pm 0.2$ & $2.0 \pm 0.0$ & $2.3 \pm 0.2$ & $2.4 \pm 0.2$ & $2.8 \pm 0.2$ & $39.6 \pm 12.3$ & $39.6 \pm 8.5$ & $40.0 \pm 3.6$ \\
\hline Mevrouw Gerard Kint & 0 & $0.9 \pm 0.1$ & $1.1 \pm 0.1$ & $1.8 \pm 0.2$ & $2.4 \pm 0.2$ & $2.7 \pm 0.2$ & $13.3 \pm 5.2$ & $9.7 \pm 0.3$ & $15.0 \pm 7.2$ \\
\hline Mevrouw Marc Van Eetvelde & 0 & $0.6 \pm 0.2$ & $1.2 \pm 0.2$ & $1.6 \pm 0.3$ & $2.2 \pm 0.2$ & $2.2 \pm 0.3$ & $50.3 \pm 11.7$ & $9.0 \pm 7.5$ & $21.6 \pm 20.7$ \\
\hline Michelle Marie & $0.2 \pm 0.2$ & $1.6 \pm 0.2$ & $2.6 \pm 0.2$ & $3.0 \pm 0.2$ & $3.4 \pm 0.2$ & $3.6 \pm 0.2$ & $14.3 \pm 0.9$ & $22.0 \pm 3.8$ & $7.3 \pm 4.3$ \\
\hline Mistral & 0 & 0 & 0 & $0.2 \pm 0.2$ & $0.8 \pm 0.2$ & $0.6 \pm 0.2$ & $9.7 \pm 8.2$ & 0 & $7.3 \pm 4.3$ \\
\hline Mont Blanc & $0.1 \pm 0.1$ & $1.4 \pm 0.2$ & $2.4 \pm 0.2$ & $2.8 \pm 0.2$ & $3.0 \pm 0.2$ & $3.1 \pm 0.1$ & $36.3 \pm 17.0$ & $29.6 \pm 4.8$ & $43.6 \pm 13.6$ \\
\hline Noralinde & $0.6 \pm 0.3$ & $1.9 \pm 0.1$ & $2.7 \pm 0.2$ & $2.9 \pm 0.1$ & $3.0 \pm 0.0$ & $3.3 \pm 0.2$ & $32.0 \pm 6.0$ & $35.0 \pm 15.4$ & $27.0 \pm 11.3$ \\
\hline Nordlicht & $0.3 \pm 0.2$ & $1.4 \pm 0.2$ & $2.8 \pm 0.2$ & $2.9 \pm 0.1$ & $3.0 \pm 0.0$ & $3.0 \pm 0.0$ & $19.3 \pm 1.2$ & $45.3 \pm 18.8$ & $52.6 \pm 14.3$ \\
\hline Otto & 0 & $1.2 \pm 0.2$ & $2.2 \pm 0.2$ & $3.0 \pm 0.0$ & $3.1 \pm 0.2$ & $3.7 \pm 0.2$ & $21.3 \pm 8.2$ & $49.3 \pm 21.1$ & $63.3 \pm 34.9$ \\
\hline Prinses Maria Pia & 0 & $1.3 \pm 0.2$ & $1.7 \pm 0.2$ & $2.7 \pm 0.2$ & $2.8 \pm 0.2$ & $3.0 \pm 0.0$ & $38.6 \pm 29.8$ & $57.3 \pm 9.8$ & $36.3 \pm 14.5$ \\
\hline Roxette & $0.1 \pm 0.1$ & $1.4 \pm 0.2$ & $2.8 \pm 0.2$ & $3.0 \pm 0.0$ & $3.0 \pm 0.0$ & $3.1 \pm 0.1$ & $46.3 \pm 21.0$ & $44.6 \pm 5.2$ & $57.3 \pm 18.2$ \\
\hline Sachsenstern & 0 & $0.2 \pm 0.2$ & $0.7 \pm 0.2$ & $1.0 \pm 0.2$ & $2.0 \pm 0.2$ & $2.1 \pm 0.1$ & $17.6 \pm 2.9$ & $11.3 \pm 4.9$ & $11.3 \pm 2.9$ \\
\hline Tamira & 0 & $1.2 \pm 0.2$ & $1.4 \pm 0.2$ & $2.4 \pm 0.2$ & $3.0 \pm 0.2$ & $3.2 \pm 0.2$ & $17.6 \pm 5.8$ & $25.0 \pm 3.1$ & $26.6 \pm 10.7$ \\
\hline Thesla & 0 & $1.1 \pm 0.1$ & $1.4 \pm 0.2$ & $2.3 \pm 0.2$ & $2.8 \pm 0.2$ & $3.0 \pm 0.0$ & $25.0 \pm 9.2$ & $12.0 \pm 5.1$ & $23.0 \pm 7.2$ \\
\hline Yankadi & 0 & $1.1 \pm 0.1$ & $2.0 \pm 0.0$ & $2.7 \pm 0.2$ & $2.8 \pm 0.2$ & $2.9 \pm 0.1$ & $34.3 \pm 23.5$ & $81.0 \pm 16.6$ & $53.6 \pm 26.7$ \\
\hline Desiree & $0.1 \pm 0.1$ & $1.0 \pm 0.0$ & $2.0 \pm 0.2$ & $2.6 \pm 0.2$ & $2.9 \pm 0.1$ & $2.9 \pm 0.1$ & $22.6 \pm 3.8$ & $30.6 \pm 12.5$ & $11.6 \pm 1.2$ \\
\hline Nazarena & $0.3 \pm 0.2$ & $1.3 \pm 0.2$ & $2.3 \pm 0.2$ & $2.3 \pm 0.2$ & $2.8 \pm 0.2$ & $2.7 \pm 0.2$ & $14.6 \pm 6.3$ & $15.0 \pm 1.5$ & $25.3 \pm 12.4$ \\
\hline Fragrantissimum & 0 & 0 & 0 & $0.1 \pm 0.1$ & $0.6 \pm 0.3$ & $0.3 \pm 0.2$ & $16.0 \pm 3.8$ & $2.3 \pm 1.3$ & $91.6 \pm 29.7$ \\
\hline R. burmanicum (Yellow) & 0 & 0 & $1.0 \pm 0.2$ & $1.3 \pm 0.2$ & $1.8 \pm 0.2$ & $1.8 \pm 0.2$ & $14.6 \pm 2.9$ & $54.6 \pm 21.5$ & $49.6 \pm 2.9$ \\
\hline NA 40246 KURIO & $0^{\mathrm{a}}$ & $0.3 \pm 0.2^{a}$ & $0.9 \pm 0.2^{\mathrm{a}}$ & $1.0 \pm 0.0^{\mathrm{a}}$ & $1.7 \pm 0.2^{\mathrm{a}}$ & $2.1 \pm 0.2^{\mathrm{a}}$ & $16.6 \pm 3.5$ & $6.7 \pm 3.3^{b}$ & $18.0 \pm 8.3$ \\
\hline
\end{tabular}

a Average damage rate based on eight observations.

b Average number of broad mites per shoot tip based on two samples. 
Table 4: Two-way-ANOVA indicating the effect of different time intervals and Rhododendron cultivars on the given damage rates and the average counted number of broad mites in experiments 1 and 2 . In experiment 1 the time intervals for damage rate were $7,14,21,28,35,42$ and 49 dpi. The time intervals for broad mite counts were 21 and $49 \mathrm{dpi}$. Time intervals for experiment 2 were 8, 15, 22, 29, 36 and 43 dpi and 15, 29 and 43 dpi, for damage rate and broad mite counts, respectively.

\begin{tabular}{|c|c|c|c|c|}
\hline \multirow[t]{2}{*}{ Source } & \multicolumn{2}{|c|}{ Experiment 1} & \multicolumn{2}{|c|}{ Experiment 2} \\
\hline & $\begin{array}{l}\text { Broad } \\
\text { mites }\end{array}$ & $\begin{array}{c}\text { Damage } \\
\text { rate }\end{array}$ & $\begin{array}{l}\text { Broad } \\
\text { mites }\end{array}$ & $\begin{array}{c}\text { Damage } \\
\text { rate }\end{array}$ \\
\hline \multicolumn{5}{|c|}{ Time interval } \\
\hline $\mathrm{F}$ & 21.51 & 235.82 & 2.47 & 1245.41 \\
\hline $\mathrm{df}$ & 1 & 6 & 2 & 5 \\
\hline$p$ & $<0.001$ & $<0.001$ & 0.088 & $<0.001$ \\
\hline \multicolumn{5}{|l|}{ Cultivar } \\
\hline $\mathrm{F}$ & 7.24 & 21.06 & 6.58 & 126.21 \\
\hline$d f$ & 29 & 29 & 29 & 29 \\
\hline$p$ & $<0.001$ & $<0.001$ & $<0.001$ & $<0.001$ \\
\hline \multicolumn{5}{|c|}{ Time interval $\times$ cultivar } \\
\hline $\mathrm{F}$ & 2.74 & 1.85 & 1.55 & 5.74 \\
\hline $\mathrm{df}$ & 29 & 174 & 58 & 145 \\
\hline$p$ & $<0.001$ & $<0.001$ & 0.015 & $<0.001$ \\
\hline \multicolumn{5}{|l|}{ Error } \\
\hline df & 112 & 510 & 179 & 1434 \\
\hline
\end{tabular}

Pink', 'Nordlicht', 'Michelle Marie', 'Sachsenstern', 'Mevrouw Gerard Kint' and 'Elien' was based on the different characteristics and the differences in patterns of broad mite counts and damage rates in experiment 1 and 2 . The outcome of this experiment was comparable with that of experiments 1 and 2. The final average damage rate for cultivars 'Aiko Pink', 'Nordlicht' and 'Michelle Marie' was higher than 2.4. 'Elien' had the lowest average damage rate $(<0.5)$. The damage rates for 'Sachsenstern' and 'Mevrouw Gerard Kint' were around 1.5 at $56 \mathrm{dpi}$. The damage rates for 'Elien' did not differ significantly at the various time intervals (Fig. 1 and Table 5). In contrast, damage rates varied significantly among 'Aiko', 'Mevrouw Gerard Kint', 'Nordlicht', 'Sachsenstern' and 'Michelle Marie' at the different time intervals (Fig. 1 and Table 5). Also the number of broad mites counted at the different time intervals was significantly different for 'Aiko', 'Mevrouw Gerard Kint', 'Nordlicht', 'Sachsenstern' and 'Michelle Marie'. At the same time intervals, broad mite counts did not differ significantly for 'Elien' (Fig. 1 and Table 5). Approximately the same number of broad mites was counted on all six cultivars 14 and 28 days after the initial infestation, indicating that a homogenous spread of mites was obtained after the reinfestation at day 12; however, at the end of the experiment, mites were no longer detected on 'Elien'. On 'Michelle Marie', 'Sachsenstern' and 'Mevrouw Gerard Kint' a comparable number of ca. 13 broad mites per shoot tip were counted, while the highest numbers of broad mites were observed on 'Aiko Pink' and 'Nordlicht' at the end of the experiment. On the latter cultivars, broad mite numbers approximately doubled during the last two time intervals, reaching around 35 broad mites per shoot tip. On 'Aiko Pink' and 'Nordlicht' a higher number of immature life stages was found than in the other cultivars. The number of eggs, lar-

Table 5: Repeated calculations of ANOVA indicating the effect of time intervals on the given damage rates and the average counted number of broad mites on 'Aiko', 'Mevrouw Gerard Kint', 'Nordlicht', 'Sachsenstern', 'Elien' or 'Michelle Marie' in experiment 3. Time intervals for broad mite counts and damage rates were 7, 14, 21, 28, 35, 42, 49 and 56 dpi.

\begin{tabular}{|c|c|c|c|c|c|c|}
\hline Source & 'Aiko' & 'Mevrouw Gerard Kint' & 'Nordlicht' & 'Sachsenstern' & ‘Elien’ & 'Michelle Marie' \\
\hline \multicolumn{7}{|c|}{ Broad mites } \\
\hline $\mathrm{F}$ & 6.07 & 6.07 & 14.11 & 17.97 & 2.14 & 16.62 \\
\hline df & 3 & 3 & 3 & 3 & 3 & 3 \\
\hline$p$ & 0.003 & 0.003 & $<0.001$ & $<0.001$ & 0.122 & $<0.001$ \\
\hline \multicolumn{7}{|l|}{ Error } \\
\hline df & 24 & 24 & 24 & 24 & 24 & 24 \\
\hline \multicolumn{7}{|c|}{ Damage rates ${ }^{\mathrm{a}}$} \\
\hline $\mathrm{F}$ & 67.31 & 31.47 & 39.42 & 27.86 & 1.27 & 53.19 \\
\hline df & 2.31 & 2.73 & 3.33 & 2.81 & 2.06 & 2.34 \\
\hline$p$ & $<0.001$ & $<0.001$ & $<0.001$ & $<0.001$ & 0.309 & $<0.001$ \\
\hline \multicolumn{7}{|l|}{ Error } \\
\hline df & 18.45 & 21.83 & 26.64 & 22.47 & 16.50 & 26.74 \\
\hline
\end{tabular}

a Mauchly's test of sphericity indicated that the assumption had been violated for each cultivar $(p<0.05)$. Degrees of freedom (df) were corrected using the Greenhouse-Geisser correction. 

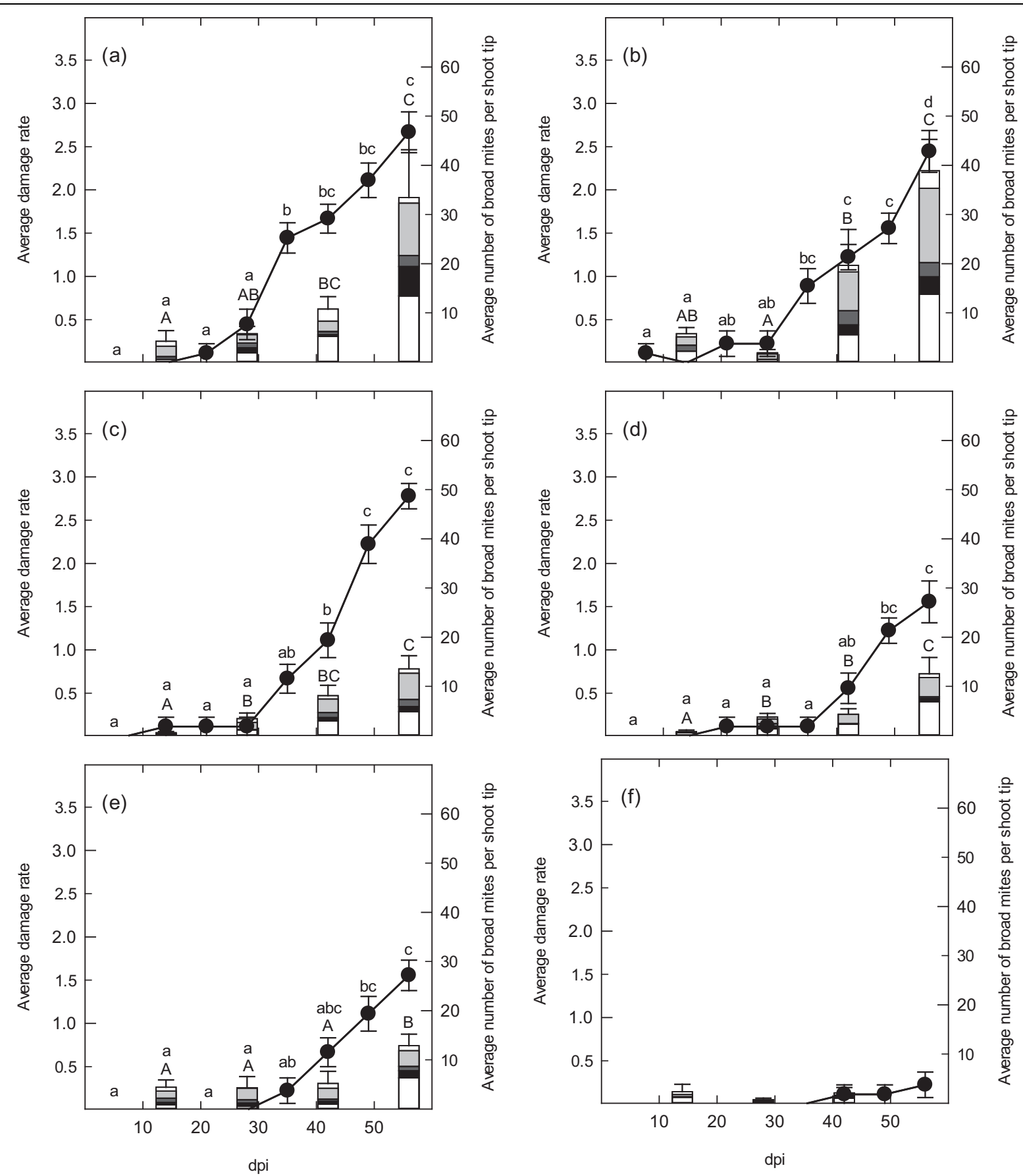

Fig. 1: Average damage rates (line) and number of broad mites per shoot tip (bars) observed for R. simsii hybrids (a) 'Aiko Pink', (b) 'Nordlicht', (c) 'Michelle Marie', (d) 'Sachsenstern', (e) ‘Mevrouw Gerard Kint' and (f) 'Elien' during experiment 3. Female broad mites (open bar), male broad mites (black bar), nymphs (dark gray bar), larvae (light gray bar) and eggs (open bar) are shown. Data are means \pm SE, $n=9$ for damage rates and broad mite counts. Different letters in lower case (damage rates) and in upper case (broad mite counts) indicate significant differences between the time intervals for each cultivar.

vae and nymphs increased after every sampling on these two cultivars (Fig. 1).

\section{Discussion}

In the present study we applied artificial infestation by placing infested $H$. helix plants around the plants of the tested cultivars. From preliminary experiments it was clear that the number of broad mites remains stable on the infestation source at different time intervals. This continually-present artificial infestation source resulted in an infestation of all cultivars at the start of each experiment (Table 2, Table 3 and Fig. 1). During the experiments, we observed much variation between the cultivars in the average broad mite counts and damage rates. In both experiment 1 and 2, 'Mistral' and 
its color sport 'Elien' were tolerant to broad mite. Hence, few broad mites were found and damage rates were low. 'Bergmann Feu', 'Doctor Bergmann Albus' and 'Madame Charles Gabert', which are all bud sports of the 'Doctor Bergmann' series (Table 1), responded in the same way and were all susceptible to broad mite infestations, as shown by high damage rates and large numbers of broad mites. The screening of cultivars randomly placed in a plot, as done in experiment 1 and 2, might lead to a bias when these cultivars with an unknown susceptibility for broad mites have a different attractiveness to the mites. Therefore, in experiment 3 each of the six selected cultivars was screened in an individual plot. Nonetheless, the patterns observed for both the average damage rates and the mite counts of the six cultivars tested in experiment 3 were comparable with the results obtained for the same cultivars in the first two screening experiments. This suggests that placing cultivars randomly in a plot does not affect the outcome of the experiments. Furthermore, these findings suggest that the artificial infestation using $H$. helix as a carrier for the mites is an appropriate method for conducting screenings on $R$. simsii hybrids in the greenhouse.

The collection of 32 Rhododendron cultivars, mainly containing $R$. simsii hybrids, showed a wide genetic variation in susceptibility against broad mites, as evidenced by the observed differences in mite counts and damage rates. Although significant correlations between damage rates and broad mite counts were found, a direct link between both observations was not obvious when checked at the level of individual cultivars. In a previous study, no significant correlations between broad mite counts and levels of damage were observed on cucumber, Cucumis sativus (Grinberg et al. 2005). In contrast, significant positive correlations between ratings of outgrowing terminals and broad mite counts in watermelon were reported by Kousik et al. (2007). A positive correlation between broad mite growth rate and injury was found on physic nut, Jatropha curcas (Evaristo et al. 2013).

On Capsicum (Echer et al. 2002) and watermelon (Kousik et al. 2007) broad mites have a tendency to aggregate, which leads to a high spatial variability of mite populations on one plant. This tendency might be responsible for the unclear relationship between broad mite counts and damage rates in the $R$. simsii hybrids. To account for this we transformed broad mite counts by taking the natural logarithm of the actual broad mite count plus one. Kousik et al. (2007) applied a log-transformation of the mite counts before further statistical analysis. Another way to tackle this problem would be to increase the number of samples taken on the plant, but this might interfere with the damage rating process of monitored plants in the long term. Indeed, the more samples taken on one entry, the less shoots that remain, inhibiting proper evaluation of this entry. A second factor that could distort the correlation between broad mite counts and the damage rate is the difference among cultivars in the emergence of symptoms caused by a given density of broad mites. It has been reported that injury symptoms can appear very rapidly on some hosts with only a few mites, resulting in symptoms that are severe enough to cause economic dam- age (Dhooria \& Bhindra 1977, Basset 1981, de Coss-Romero $\&$ Peña 1998). Results from the present study indicate different types of responses to a broad mite infection. At $43 \mathrm{dpi}$ in experiment 2, 'Elien' showed nearly no symptoms (average damage rate of 0.6 ) and no broad mites were detected (Table 3). On the other hand, 'Aiko Pink' and 'Nordlicht' showed high damage rates (3.0 for both cultivars) and high mite counts ( $>50$ mites on average), at the same time interval as in experiment 2 (Table 3 ). In contrast, a high damage rate (3.6) was noted for 'Michelle Marie' but only a few mites (seven on average) were found on that azalea under the same conditions (Table 3 ). However in the last case, on average 22 broad mites were found two weeks earlier at 29 dpi in experiment 2 (Table 3). In Capsicum genotypes, the assessment of symptoms caused by broad mites was shown to be more suitable for separating the accessions in resistant or susceptible types (Echer et al. 2002). For ornamental plants, however, the entire plant has economic value; and only undamaged or uninfested plants are marketable. Evaluation of the susceptibility of an accession of pot azalea should therefore initially be based on the damage rate.

At a constant temperature of $20^{\circ} \mathrm{C}$, broad mite eggs are able to reach adulthood in 6.6 days on $R$. simsii hybrid 'Nordlicht' (Luypaert et al. 2014). Development at higher temperatures is achieved even more quickly, e.g. in 4.2 days at $25^{\circ} \mathrm{C}$ and 3.5 days at $30^{\circ} \mathrm{C}$ (Luypaert et al. 2014). The present experiments were conducted at a minimum temperature of $21^{\circ} \mathrm{C}$. Broad mites could thus have completed two to three generations during the two consecutive recording times during experiment 3. Nevertheless, on 'Elien', which was a tolerant cultivar based on the damage rates, no mites were found at the end of the experiment (Fig. 1). This re-confirmed the tolerant character of that cultivar. On 'Aiko Pink' and 'Nordlicht', two very susceptible cultivars, the mite population rose over time. Total mite counts increased at every subsequent sampling interval (Fig. 1). In addition to the number of adult males and females, the number of eggs, larvae and nymphs increased, demonstrating the mite's affinity for these cultivars. Adult females were found more frequently than males, which is in line with the natural adult male:female ratio averaging 1:4 (Gerson 1992) (Fig. 1). Due to only two recording times for broad mite counting it is not appropriate to discuss the mite's population development in experiment 1 . In experiment 2 , total mite populations remained either stable or increased over time in susceptible cultivars. However, on 'Lara', a highly sensitive cultivar, a rapid decline of the mite population was observed between 29 and 43 dpi. Under optimal conditions, broad mite populations may increase to large numbers and then rapidly decline due to the deterioration of food quality and of weather conditions or to the presence of natural enemies (Gerson 1992). Since weather conditions in our study did not change and no predatory mites were present during the experiments, it is suggested that the mite population declined as a result of lowered host plant quality, as evidenced by bronzed growing shoot tips, hard and necrotic leaf tissue and severe curling of leaves.

The factors underlying tolerance against broad mites in the above-evaluated cultivars of pot azalea are unknown. 
Thungrabeab \& Boonlertnirun (2002) and Matos et al. (2009) reported on the effect of trichomes as a limiting trait for the development of broad mites in Capsicum species. Tolerance to damage caused by broad mites was observed in $S$. polyadenium and in seedlings of $S$. tarijense and $S$. berthaultii, which had foliar hairs with a sticky tip (Gibson \& Valencia 1978). The tolerance characteristics of cultivars 'Mistral' and 'Elien' in our study might be related to the presence of this type of foliar glandular hairs. However, 'Marc Van Eetvelde' and 'Lara', two R. simsii hybrids that also have foliar glandular hairs, were susceptible to broad mites. Regarding the parentage of these four cultivars, it is remarkably that 'Mistral', 'Marc Van Eetvelde' and 'Lara' resulted from a cross with 'Heiwa-no-hikari' as one of the parents (Table 1). Since 'Elien' is a sport from 'Mistral' it is also related to 'Heiwa-no-hikari'. Ahmed et al. (2001) examined numerous biochemical constituents in 15 C. annuum cultivars. Tannins, phenols, potassium, calcium, magnesium and chlorophyll had a negative correlation with broad mite incidence. In contrast, total sugars, protein content and nitrogen content were correlated positively with a broad mite infestation. The authors found no correlation with the phosphorous content of the leaves. The assessment of several of these compounds in a selection of susceptible versus tolerant $R$. simsii hybrid cultivars might provide extra information regarding the traits that account for tolerance in azalea against broad mites.

In conclusion, the present study has demonstrated that the use of a continually present artificial infestation source is an appropriate method to screen Rhododendron spp. for susceptibility to broad mites. Significant genetic variation was found within a selection of $R$. simsii hybrids. Among the evaluated cultivars, 'Emil De Coninck' and 'Mont Blanc' were the most susceptible, whereas 'Mistral' and its sport 'Elien' proved to be tolerant towards broad mites. These findings may be useful to develop further breeding for tolerance against these mites in $R$. simsii hybrids. Further research will be necessary to understand factors underlying the observed tolerance.

\section{Acknowledgements}

The authors wish to thank the Research Centre for Ornamental Plants in Destelbergen, Belgium, for maintaining and providing a broad mite culture on Hedera helix. We are also grateful to the ILVO greenhouse technicians for excellent plant cultivation and Magali Losschaert for outstanding technical assistance during the experiments. This research was funded by the Institute for the Promotion of Innovation through Science and Technology in Flanders (IWT-Vlaanderen Grant No. 100859).

\section{References}

Ahmed K, Rao VH \& Rao PP, 2001. Resistance in chilli cultivars to yellow mite Polyphagotarsonemus latus Banks. Indian J Agric Res 35, 5-99.
Basset P, 1981. Observations on broad mite (Polyphagotarsonemus latus) (Acarina: Tarsonemidae) attacking cucumber. Proc Br Crop Prot Conf Pests Dis 1, 99-103.

Commission Directive 2005/864/EC concerning the noninclusion of endosulfan in Annex I to Council Directive 91/414/EEC and the withdrawal of authorization for plant protection products containing this active substance [2005] OJ L 317/25.

de Coss-Romero M \& Peña JE, 1998. Relationship of broad mite (Acari: Tarsonemidae) to host phenology and injury levels in Capsicum annuum. Fla Entomol 81, 515-526.

Desai HR, Bandhania KA, Patel AJ, Patel MB \& Rai AB, 2006. Screening of chilli varieties/germplasms for resistance to yellow mite, Polyphagotarsonemus latus Banks in South Gujarat. Pest Management in Horticultural Ecosystems 12, 55-62. Desai HR, Bandhania KA, Rai AB, Patel AJ \& Patel MB, 2007. Assessment of yield loss and resistance to yellow mite Polyphagotarsonemus latus Banks in chilli. Veg Sci 31, 46-50.

Dhooria MS \& Bhindra OS, 1977. Polyphagotarsonemus latus (Banks) a mite pest of chilli and potato in Punjab. Acarol Newsl 4, 7-9.

Directive of the European parliament and the council 2009/ 128 /EC establishing a framework for community action to achieve the sustainable use of pesticides [2009] OJ L309/71.

Echer MM, Fernandes MCA, Ribeiro RLD \& Peracchi AL, 2002. Avaliação de genótipos de Capsicum para resistência ao ácaro branco. Horticultura Brasileira 20, 217-221.

Evaristo AB, Venzon M, Matos FS, de Freitas RG, Kuki KN \& Dias LAS, 2013. Susceptibility and physiological responses of Jatropha curcas accessions to broad mite infestation. Exp Appl Acarol 60, 485-496.

Ewing HE, 1939. A revision of the mites of the subfamily Tarsoneminae of North America, the West Indies and the Hawaiian Islands. USDA Tech Bull 653, USA.

Gerson U, 1992. Biology and control of the broad mite, Polyphagotarsonemus latus (Banks) (Acari: Tarsonemidae). Exp Appl Acarol 13, 163-178.

Gibson RW \& Valencia L, 1978. A survey of potato species for resistance to the mite Polyphagotarsonemus latus, with particular reference to the protection of Solanum berthaultii and $S$. tarijense by glandular hairs. Potato Res 21, 217-223.

Gobin B, Audenaert J, Vissers M, Van Delsen B, Vlaeminck M \& Pauwels E, 2013. Broad mite control in woody ornamentals: developing an integrated pest management solution. Acta Horticulturae 990: II International symposium on woody ornamentals of the moderate zone (ed. by J. Van Huylenbroeck, M.C. Van Labeke \& K. Van Laere). ISHS, Leuven, Belgium, pp. 47-53.

Gobin B, Vissers M \& Pauwels E, 2011. Integrated Pest Management solutions for the control of Polyphagotarsonemus latus in ornamentals: from trial to practice. IOBC/WPRS Bull 68, 47-50.

Grinberg M, Perl-Treves R, Palevsky E, Shomer I \& Soroker V, 2005. Interaction between cucumber plants and the broad mite, Polyphagotarsonemus latus: From damage to defense gene expression. Entomol Exp Appl 115, 135-144.

Heungens A, 1986. Soft-skinned mites in azalea culture and comparable control results on other host plants. Verbondsnieuws voor de Belgische Sierteelt 30, 257-269. 
Heursel J, 1999. Azalea's oorsprong, veredeling en cultivars. Lannoo-Terra, Tielt, Belgium, 192 pp.

Jana SK, Nair N, Sarkar PK \& Somchoudhury AK, 2007. Varietal susceptibility of jute varieties against yellow mite, Polyphagotarsonemus latus Banks (Acari: Tarsonemidae). J Entomol Res 31, 29-32.

Jeppson LR, Keifer HH \& Baker EW, 1975. Mites injurious to economic plants. University of California, Berkeley, 614 pp.

Kousik CS, Shepard BM, Hassell R, Levi A \& Simmons AM, 2007. Potential sources of resistance to broad mites (Polyphagotarsonemus latus) in watermelon germplasm. HortScience 42, 1539-1544.

Kulkarni SK, GastiVD, Mulge R, Madalageri MB, Kulkarni MS \& Shirol AM, 2011. Reaction of chilli genotypes against mites, [Polyphagotarsonemus latus (Banks)] and thrips, [Scirthothrips dorsalis (Hood)] under natural conditions. Karnataka J Agric Sci 24, 258-259.

Łabanowski G \& Soika G, 2006. Tarsonemid mites on ornamental plants in Poland: new data and an overview. Biological Lett 43, 341-346.

Leslie CA, 2004. The international Rhododendron register \& checklist. Second Edition. Royal Horticultural Society, London, UK, 1544 pp.
Luypaert G, Witters J, Van Huylenbroeck J, Maes M, De Riek J \& De Clercq P, 2014. Temperature-dependent development of the broad mite Polyphagotarsonemus latus (Acari: Tarsonemidae) on Rhododendron simsii. Exp Appl Acarol 6, 389-400.

Matos CHC, Pallini A, Venzon M, De Freitas RCP, Rezende DDM \& Schoereder JH, 2009. Os Tricomas de Capsicum spp. interferem nos aspectos biológicos do ácaro-branco, Polyphagotarsonemus latus Banks (Acari: Tarsonemidae)? Neotrop Entomol 38, 589-594.

Rodríguez H, Montoya A, Miranda I, Rodríguez Y \& Ramos M, 2011. Influence of the phenological phase of two pepper cultivars on the behaviour of Polyphagotarsonemus latus (Banks). Rev Proteccíon Veg 26, 73-79.

Thungrabeab M \& Boonlertnirun K, 2002. Selection for resistant varieties of pepper (Capsicum spp.) to Polyphagotarsonemus latus. Proc 19th Rajamangala University Technol Conf. pp. 157-158.

Vichitbandha P \& Chandrapatya A, 2011. Broad mite effects on chilli shoot damage and yields. Pakistan J Zool 43, 637-649.

Zhang Z-Q, 2003. Mites of greenhouses: Identification, Biology and Control. CABI, Wallingford, UK, 244 pp. 